\title{
Total Pelvic Exenteration for Primary and Recurrent Malignancies
}

\author{
F. T. J. Ferenschild $\cdot$ M. Vermaas $\cdot$ C. Verhoef $\cdot$ \\ A. C. Ansink - W. J. Kirkels · A. M. M. Eggermont • \\ J. H. W. de Wilt
}

Published online: 7 May 2009

(c) The Author(s) 2009. This article is published with open access at Springerlink.com

\begin{abstract}
Introduction Complete resection is the most important prognostic factor in surgery for pelvic tumors. In locally advanced and recurrent pelvic malignancies, radical margins are sometimes difficult to obtain because of close relation to or growth in adjacent organs/structures. Total pelvic exenteration (TPE) is an exenterative operation for these advanced tumors and involves en bloc resection of the rectum, bladder, and internal genital organs (prostate/ seminal vesicles or uterus, ovaries and/or vagina).

Methods Between 1994 and 2008, a TPE was performed in 69 patients with pelvic cancer; 48 with rectal cancer $(32$ primary and 16 recurrent), 14 with cervical cancer ( 1 primary and 13 recurrent), 5 with sarcoma ( 3 primary and 2 recurrent), 1 with primary vaginal, and 1 with recurrent endometrial carcinoma. Ten patients were treated with neoadjuvant chemotherapy and 66 patients with preoperative radiotherapy to induce down-staging. Eighteen patients received IORT because of an incomplete or marginal complete resection.
\end{abstract}

F. T. J. Ferenschild $(\bowtie) \cdot$ M. Vermaas · C. Verhoef .

A. M. M. Eggermont - J. H. W. de Wilt

Department of Surgical Oncology, Erasmus MC-Daniel den

Hoed Cancer Center, P.O. Box 5201, 3008 AE Rotterdam,

The Netherlands

e-mail: floferenschild@kpnplanet.nl

J. H. W. de Wilt

e-mail: j.h.w.dewilt@erasmusmc.nl

A. C. Ansink

Department of Gynaecology, Erasmus MC-Daniel den Hoed Cancer Center, Rotterdam, The Netherlands

W. J. Kirkels

Department of Urology, Erasmus MC-Daniel den Hoed Cancer Center, Rotterdam, The Netherlands
Results The median follow-up was 43 (range, 1-196) months. Median duration of surgery was 448 (range, 300670) minutes, median blood loss was 6,300 (range, 75021,000) $\mathrm{ml}$, and hospitalization was 17 (range, 4-65) days. Overall major and minor complication rates were $34 \%$ and $57 \%$, respectively. The in-hospital mortality rate was $1 \%$. A complete resection was possible in $75 \%$ of all patients, a microscopically incomplete resection (R1) in $16 \%$, and a macroscopically incomplete resection (R2) in 9\%. Fiveyear local control for primary locally advanced rectal cancer, recurrent rectal cancer, and cervical cancer was $89 \%$, 38\%, and 64\%, respectively. Overall survival after 5 years for primary locally advanced rectal cancer, recurrent rectal cancer, and cervical cancer was $66 \%, 8 \%$, and $45 \%$.

Conclusions Total pelvic exenteration is accompanied with considerable morbidity, but good local control and acceptable overall survival justifies the use of this extensive surgical technique in most patients, especially patients with primary locally advanced rectal cancer and recurrent cervical cancer.

\section{Introduction}

Locally advanced pelvic tumors without distant metastases can cause severe local problems, such as pain, voiding, and defecation problems, and may result in a decreased quality of life $[1,2]$. Extensive surgery often is the only possibility for complete resection, thereby attempting to provide local control and palliation. In case of involvement of the base or trigone of the bladder or the prostate, a total pelvic exenteration (TPE) with resection of the rectum together with bladder, lower ureters, and internal genital organs could 
potentially salvage the patient [3]. After resection of the bladder, an ileal conduit is usually constructed according to the technique described by Bricker [4]. TPE has been performed in primary or recurrent cancer of the cervix, rectum, vagina, uterine corpus, vulva, prostate, bladder, and for pelvic sarcoma $[5,6]$.

Since the introduction of the technique by Brunswick in 1948 , the initially poor quality of life and high mortality and morbidity associated with the technique have substantially improved [7-9]. However, morbidity after this extensive surgical procedure is still high and reported to be between $13 \%$ and 64\% [10-12]. Five-year survival rates after TPE for patients with primary disease range between $32 \%$ and $66 \%$ and in patients with recurrent disease from $0 \%$ to $23 \%$ $[3,7,12]$. Hence, a careful selection in a multidisciplinary setting of patients is of paramount importance.

In the present study, all patients who underwent a pelvic exenteration were reviewed. Preoperative morbidity and mortality, local recurrence, disease-free, and overall survival rates were studied and prognostic factors for local control or survival were analyzed.

\section{Patients and methods}

In the Erasmus University Medical Center-Daniel den Hoed Cancer Center, 69 TPEs were performed during the period from 1994 to 2008 for locally advanced primary or locally recurrent pelvic malignancies. Forty-eight patients with rectal cancer (32 primary locally advanced and 16 recurrent), 14 with cervical cancer (1 primary and 13 recurrent), 5 with pelvic sarcoma (3 primary and 2 recurrent), 1 with primary vagina carcinoma, and 1 with recurrent endometrial carcinoma. Fifty patients were men, with a median age of 61 (range, 30-76) years.

Patients were preoperatively analyzed and selected using CT or MRI scanning, or both, of the small pelvis (90\% and 59\%, respectively). On indication, a cystoscopy was performed to rule out or establish bladder involvement. Screening for distant metastases was performed using thoracic and abdominal CT scan in all patients.

All 32 patients with primary rectal cancer received 50 Gy preoperative (chemo)radiation. Thirteen of 26 patients (10 cervical, 2 sarcoma, and 1 rectal cancer patient) with recurrent cancer previously received radiotherapy during treatment of the primary tumor (median dosage, 50 (range, 46-60) Gy. One patient was re-irradiated (27 Gy) after 50 Gy adjuvant radiation after primary treatment for rectal cancer. One patient with recurrent rectal cancer received chemotherapy as primary treatment. Primary treatment for patients with recurrent cancer is described in Table 1.

In 1997 an intraoperative radiation therapy (IORT) program was started in our hospital and the technique was
Table 1 Previous procedures of patients with recurrent pelvic cancer

\begin{tabular}{|c|c|c|}
\hline Rectal & $(n=16)$ & $\begin{array}{l}\text { Abdominal perineal resection } \\
\text { Low anterior resection } \\
\text { Rectosigmoid resection and end } \\
\text { colostomy }\end{array}$ \\
\hline Cervical & $(n=13)$ & $\begin{array}{l}\text { Radical hysterectomy } \\
\text { Extrafascial abdominal hysterectomy } \\
\text { Vaginal hysterectomy } \\
\text { Chemoradiation }\end{array}$ \\
\hline Sarcoma & $(n=2)$ & $\begin{array}{l}\text { Local excision } \\
\text { Hysterectomy }\end{array}$ \\
\hline Endometrial & $(n=1)$ & Abdominal uterus extirpation \\
\hline
\end{tabular}

described previously [13]. Briefly, IORT with HDR brachytherapy was given to patients who had a minimal circumferential-free resection margin $\leq 2 \mathrm{~mm}$. The resection margin was judged on frozen sections taken during surgery. IORT was performed using the Flexible Intraoperative Template (FIT) developed at our department, delivering a dose of $10 \mathrm{~Gy}$, usually at 1-cm depth from the applicator surface.

Pathology

All staging, except from the staging of sarcoma, was performed according to the AJCC TNM criteria, and completeness of resection was divided in R0 (complete resection of tumor), R1 (microscopic tumor remnant in circumferential margin), and R2 (macroscopically not complete resection). Recurrent rectal tumors were staged according to the Wanebo classification for recurrent tumors [14]. Since 2002, all pathology examinations for rectal cancer have been performed according to the guidelines of Quirke et al. [15].

\section{Evaluation of morbidity and mortality}

Hospital charts were studied to collect patient characteristics, operation techniques, and follow-up. Surgery-related morbidity was divided into major and minor complications. Major morbidity was defined as a complication that requires (surgical) reintervention. All other complications were classified as minor.

Statistical analysis of survival and local control

Survival time was calculated from the date of resection of the tumor until the last follow-up attendance or until death. Local control was calculated from the date of resection until the histological or evident radiological presence of a local recurrence. The cumulative survival and local control rate after surgery were calculated using the Kaplan-Meier 
method [16]. Univariate survival comparisons were executed using the log-rank test. Cox proportional hazards analysis was used for multivariate analysis of prognostic factors for local control and overall survival [17]. The level of significance was defined as $p<0.05$.

\section{Results}

At the time of diagnosis patients presented with complaints of pain $(19 \%)$, changes in defecation $(25 \%)$, changes in urinary miction $(7 \%)$, perineal pressure $(6 \%)$, and a combination of these complaints (33\%). Only seven patients $(10 \%)$ did not have complaints and were diagnosed during routine follow-up.

At the preoperative physical examination, $47 \%$ of the gynecological tumors were clinically fixed to the rectum and $74 \%$ percent of the rectal tumors were clinically fixed to the prostate and bladder.

An exploratory staging laparotomy to create a colostomy or ileostomy was performed in 15 patients (1 sarcoma, 1 recurrent cervical, 12 primary locally advanced, and 1 recurrent rectal cancer). No distant metastases were found during previous surgery and preoperative screening.

\section{Preoperative treatment}

Ten patients (14\%) received neoadjuvant chemotherapy (5 rectal cancer, 1 primary, 3 recurrent cervical, and 1 vagina carcinoma). Forty-six patients (67\%) received preoperative radiotherapy (median dosage, 50 (range, 27-67) Gy).

\section{Surgery}

The median duration of surgery was 448 (range, 300-670) minutes. The median blood loss was 6,300 (range, 750$21,000) \mathrm{ml}$. Fifty-eight patients received a small bowel as urostoma and 11 patients a colon conduit. None of the patients had liver or diffuse peritoneal metastatic disease. In all but six patients, an omentoplasty, and in five patients, an unilateral gracilis muscle transposition was used for primary pelvic reconstruction. Indirect reconstruction with gracilis transposition (one bilateral and three unilateral) was performed in three patients because of persistent perineal wounds. No flap necrosis occurred and eventually after secondary reconstruction all perineal wounds closed in a median of 86 (range, 43-304) days.

Intraoperative and postoperative treatment

IORT was applied in 18 patients (6 primary rectal, 10 recurrent rectal, 2 recurrent cervical cancer): 8 with a marginal radical (R0) resection, 9 with a microscopically irradical resection (R1), and 1 with macroscopic tumor mass (R2). One primary sarcoma and two recurrent cervical tumors were postoperatively irradiated. None of the patients received adjuvant chemotherapy.

Postoperative

TNM and Wanebo stages and completeness of resection are depicted in Table 2. All sarcomas underwent a complete resection. The TNM stage for one vagina carcinoma was T4N0 and T3N0 for the recurrent endometrial carcinoma. Both tumors were resected complete. The median postoperative hospital stay was 17 (range, 4-65) days with an increase for the recurrent tumors compared to the primary-treated tumors ( 20 vs. 14 days).

Complications are depicted in Table 3. The most common minor complications were a superficial wound infection $(34 \%)$ and pulmonary infections (8\%). Forty-eight percent of all reinterventions were performed because of complications related to the construction of the urostomy. All of these patients had received radiotherapy ( 1 in primary treatment, 9 in neoadjuvant setting, and 1 in previous treatment, intraoperatively as in adjuvant treatment).

The major and minor complication rates were not significantly different for patients treated with or without radiotherapy.

Table 2 Pathology characteristics

\begin{tabular}{|c|c|c|c|c|c|}
\hline & & \multicolumn{2}{|c|}{ Rectal } & \multicolumn{2}{|c|}{ Cervical } \\
\hline & & Prim & $\operatorname{Rec}$ & Prim & $\operatorname{Rec}$ \\
\hline \multicolumn{2}{|l|}{ Total no. } & (32) & (16) & (1) & (13) \\
\hline \multirow[t]{4}{*}{$\mathrm{T}$ stage } & T0 & - & $8 \%^{\mathrm{a}}$ & - & $9 \%$ \\
\hline & $\mathrm{T} 2$ & - & - & - & $9 \%$ \\
\hline & T3 & $35 \%$ & $25 \%^{\mathrm{b}}$ & - & $36 \%$ \\
\hline & $\mathrm{T} 4$ & $65 \%$ & $67 \%^{\mathrm{c}}$ & $100 \%$ & $45 \%$ \\
\hline \multirow[t]{4}{*}{$\mathrm{N}$ stage } & No & $57 \%$ & $42 \%$ & $100 \%$ & $45 \%$ \\
\hline & N1 & $9 \%$ & $16 \%$ & - & - \\
\hline & $\mathrm{N} 2$ & $9 \%$ & - & - & - \\
\hline & $\mathrm{Nx}$ & $26 \%$ & $42 \%$ & - & $54 \%$ \\
\hline \multirow[t]{3}{*}{ Completeness } & R0 & $82 \%$ & $58 \%$ & $100 \%$ & $64 \%$ \\
\hline & $\mathrm{R} 1$ & $9 \%$ & $25 \%$ & - & $36 \%$ \\
\hline & R2 & $9 \%$ & $17 \%$ & - & - \\
\hline
\end{tabular}

${ }^{a}$ Wanebo classification for recurrent rectal cancer stage $\operatorname{Tr} 0$ (no recurrence)

b Wanebo classification for recurrent rectal cancer stage Tr 3 (growth into surrounding soft tissue)

c Wanebo classification for recurrent rectal cancer stage $\operatorname{Tr} 4$ (penetration anterior structures) 
Table 3 Complications and reinterventions

\begin{tabular}{|c|c|c|c|c|c|}
\hline & Prim rectum $(n=32)$ & Rec rectum $(n=16)$ & Cervix $(n=14)$ & Others $(n=7)$ & Total $(n=69)$ \\
\hline \multicolumn{6}{|l|}{ Complications } \\
\hline Minor only & $12(38 \%)$ & $5(31 \%)$ & $2(14 \%)$ & $3(43 \%)$ & $22(32 \%)$ \\
\hline Major only & - & $4(25 \%)$ & - & - & $4(6 \%)$ \\
\hline Major and minor & $9(28 \%)$ & $4(25 \%)$ & $6(43 \%)$ & $1(14 \%)$ & $20(29 \%)$ \\
\hline No complication & $11(34 \%)$ & $3(18 \%)$ & $6(43 \%)$ & $3(43 \%)$ & $23(33 \%$ \\
\hline \multicolumn{6}{|l|}{ Minor complications } \\
\hline Wound infection perineal & $4(12 \%)$ & $1(6 \%)$ & $3(21 \%)$ & $2(28 \%)$ & $10(14 \%)$ \\
\hline Wound infection midline & $6(19 \%)$ & $3(19 \%)$ & $3(21 \%)$ & $1(14 \%)$ & $13(19 \%)$ \\
\hline Pneumonia & $4(12 \%)$ & $1(6 \%)$ & $1(7 \%)$ & $1(14 \%)$ & $7(10 \%)$ \\
\hline Central venous catheter sepsis & $1(3 \%)$ & $1(6 \%)$ & - & - & $2(3 \%)$ \\
\hline Fever without known cause & $1(3 \%)$ & - & - & - & $1(1 \%)$ \\
\hline Urinary tract infection & $3(9 \%)$ & - & $2(14 \%)$ & $1(14 \%)$ & $6(9 \%)$ \\
\hline Neuropathy & $1(3 \%)$ & $1(6 \%)$ & - & - & $2(3 \%)$ \\
\hline TIA & - & $1(6 \%)$ & - & - & $1(1 \%)$ \\
\hline Decubitus & $1(3 \%)$ & $1(6 \%)$ & - & - & $2(3 \%)$ \\
\hline \multicolumn{6}{|c|}{ Major complications / required reintervention } \\
\hline \multicolumn{6}{|l|}{ Urostomy-related } \\
\hline Nefrodrain placement & $2(6 \%)$ & $2(13 \%)$ & $2(14 \%)$ & - & $6(9 \%)$ \\
\hline Small-bowel leakage-repair & $2(6 \%)$ & $1(6 \%)$ & $1(7 \%)$ & - & $4(6 \%)$ \\
\hline Reimplantation ureter & $1(3 \%)$ & - & $1(7 \%)$ & - & $2(3 \%)$ \\
\hline \multicolumn{6}{|l|}{ Other } \\
\hline Gracilis flap wound-repair & $2(6 \%)$ & - & - & - & $1(1 \%)$ \\
\hline Sartorius flap fistula repair & - & $1(6 \%)$ & - & - & $1(1 \%)$ \\
\hline Abdominal dehiscence repair & $1(3 \%)$ & - & - & - & $1(1 \%)$ \\
\hline Ileus relaparotomy & - & $1(6 \%)$ & - & - & $1(1 \%)$ \\
\hline Bleeding relaparotomy & - & - & - & $1(14 \%)$ & $1(1 \%)$ \\
\hline Abscess drainage & - & $2(12 \%)$ & $3(21 \%)$ & $1(14 \%)$ & $6(9 \%)$ \\
\hline Suture leakage repair (ileostomy) & - & - & $1(7 \%)$ & - & $1(1 \%)$ \\
\hline Enterocutaneous fistula repair & - & - & $1(7 \%)$ & - & $1(1 \%)$ \\
\hline
\end{tabular}

Major complication: a complication that causes the need for reintervention or is the cause of prolonged hospitalization

Minor complication: a complication that does not cause the need for reintervention or is the cause of prolonged hospitalization

\section{Local control}

The 5-year local control of primary rectal, recurrent rectal, and other cancer was $89 \%, 38 \%$, and 64\% (Fig. 1). All four patients with a soft-tissue sarcoma and the patients with a primary cervical tumor and primary vaginal tumor remained without local recurrence after 5 years. A recurrence at the urethra was observed 10 months after resection in the patient who was operated for a third recurrence of an endometrial tumor. The recurrence was treated with local palliative resection and the patient received systemic chemotherapy.

At univariate analysis, significant prognostic factors for an improved local control were the interval from primary resection to local recurrence more than 12 months $(p=0.012)$, type of tumor (primary rectal; $p=0.009$ ),

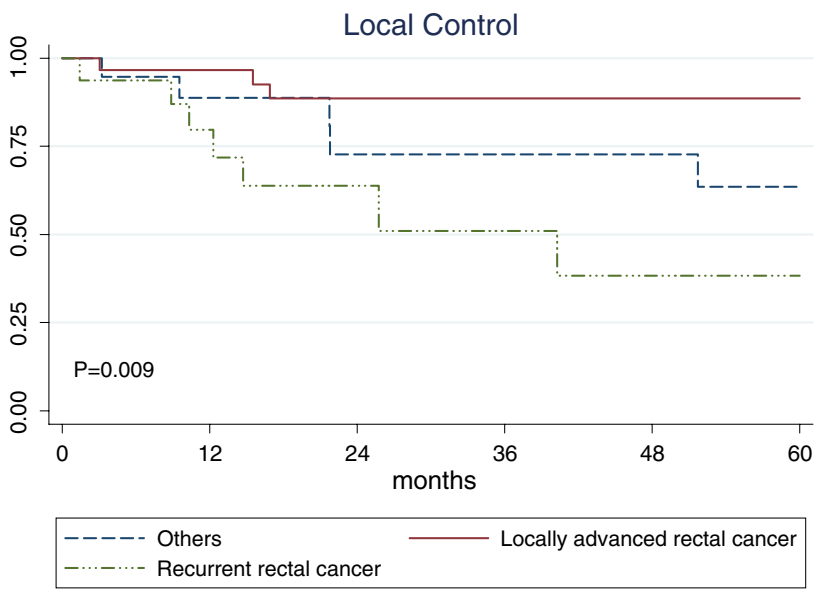

Fig. 1 Kaplan-Meier curve for local control 


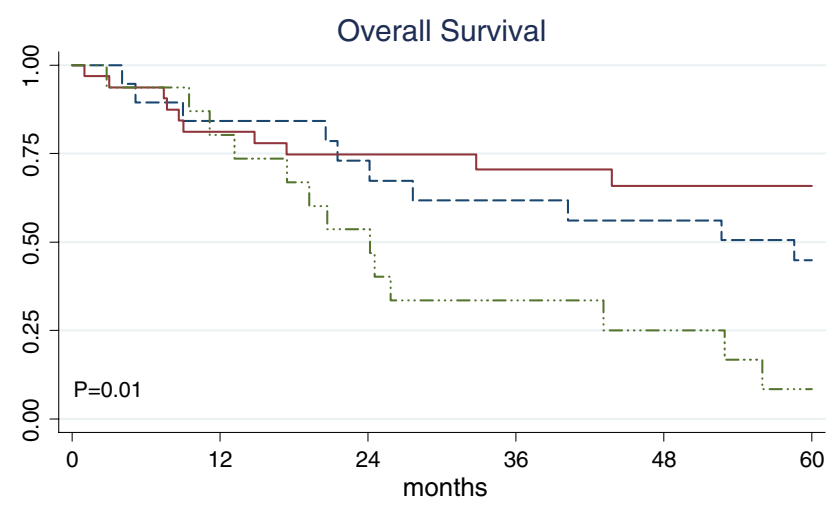

$\begin{array}{ll}---- \text { Others } & \text { Locally advanced rectal cancer } \\ -\cdots-\cdots-\text { Recurrent rectal cancer } & \end{array}$

Fig. 2 Kaplan-Meier curve for overall survival

completeness of resection $(p<0.001)$, and absence of preoperative pain $(p<0.001)$. Sex, age, preoperative radiotherapy, and positive lymph nodes did not have prognostic value on local control.

\section{Overall survival}

The 5-year overall survival of patients with primary, recurrent rectal, and other cancer was $66 \%, 8 \%$, and $45 \%$ (Fig. 2). All patients with soft-tissue sarcoma were alive after 5 years. At the last follow-up, 46 months after resection, the patient with primary cervical cancer was alive without evidence of disease. The patient with a primary vaginal tumor remained under local control but developed bone metastases 16 months after TPE and died 6 months later. The patient with the third recurrence of endometrial cancer was lost to follow-up 9 months after developing a local recurrence.

An interval from primary resection to local recurrence of more than 12 months, type of tumor (primary rectum), and completeness of resection were prognostic factors for improved survival $(p=0.01, p=0.01$, and $p<0.001$, respectively). Sex, age, preoperative radiotherapy, and positive lymph nodes did not have prognostic value on overall survival.

\section{Discussion}

The technique of total pelvic exenteration was introduced as a palliative procedure for patients with advanced gynecological cancer. Currently it is performed with curative intent in the treatment of locally advanced and recurrent pelvic disease (rectal, cervical, endometrial, vaginal, vulvar tumors, and soft-tissue sarcoma) [8]. In locally advanced tumors (without distant metastasis), complete resection is the only possibility for cure, and in patients with rectal cancer, the overall survival rate of $66 \%$ and local control rate of $89 \%$ after 5 years shows that survival with excellent local control is possible after TPE [10, 12, 13]. These results combined with the high $\mathrm{R} 0$ rate and acceptable complication rate justify the performance of TPE in this subgroup [3]. The results of TPE for recurrent rectal cancer, with local control of $38 \%$ and $8 \%$ overall survival after 5 years, indicate the poor prognosis and questionable benefit of major surgery in this group. These results are comparable with rates after nonexenterative surgery for recurrent rectal cancer and the high occurrence of distant metastasis emphasizes the importance of thorough patient selection $[5,10,12,13]$.

The majority of TPE in gynecological cancer is performed in recurrent cervical cancer [18-20]. The treatment of choice for locally advanced cervical cancer is chemoradiation. One-third of patients with primary carcinoma of the cervix will have residual disease or recurrent disease, and in up to $25 \%$ of these patients there is only local disease without systemic metastases [21]. The treatment for recurrent cervical cancer can be chemoradiation, and in a selected population, without distant metastases, exenterative surgery (posterior, anterior, or total exenteration). However, this extensive, disfiguring procedure with a relatively high complication rate should only be performed in highly motivated patients. The 5-year overall survival after treatment of recurrent cervical cancer of $45 \%$ in the present study compares to rates reported in the last decade varying from $24-54 \%$ [18, 19, 22]. This also holds true for local recurrence-free rate of $64 \%$ after 5 years, which is comparable with rates varying from $40-78 \%$ in the literature $[18,22]$. For more uncommon tumors, case reports or small series describe different results. Barakat et al. described that surgery for recurrent endometrial cancer is associated with only $20 \%$ long-term survival and high morbidity [23]. Preoperatively irradiated patients with locally advanced vaginal cancer are appropriate candidates for TPE with $>50 \%$ survival after 5 years according to Berek et al [19].

Pelvic sarcomas are rare tumors that originate from the stroma of pelvic viscera or from the retroperitoneum. Complete resection and tumor grade are the main prognostic factors for survival [24]. The four patients with sarcomas ( 2 primary and 2 recurrent) in the present study were all completely resected and did not recur in the pelvis. The $100 \%$ overall survival without local relapse in this small cohort compares favorably to the $66 \%$ survival after 2 years, which recently described by Lopes et al [6]. Above all, surgery is the only option in these kinds of tumors because there is no clear benefit of any kind of chemotherapy.

Overall complete resection rate in the present study was $75 \%$ for all tumors. The curative potential of resection 
differs between primary and recurrent tumors. A complete resection in patients with recurrent disease was possible in $65 \%$ of the patients in the present study, which was substantially lower than for patients with a primary tumor $(85 \%)$. Complete resection is more difficult in recurrent cancer because of primary resection of visceral fascia. Successful complete resection of recurrent disease often is restricted to selected patients, for example with earlydetected or limited tumor mass. As described in previous reports, complete resection of tumor is a significant prognostic factor for local control and survival [5, 10, 12, 25]. All four patients with a macroscopically incomplete resection ( 2 primary and 2 recurrent rectal cancer) died during follow-up, with a mean survival of 13 months.

Morbidity rates reported after TPE for primary and locally recurrent rectal cancer vary from $13-78 \%[10,11,26]$. We have previously reported overall morbidity rates of $61 \%$ in primary rectal cancer (26\% major and $35 \%$ minor) and $83 \%$ in recurrent rectal cancer (50\% major and 58\% minor) [3]. Infectious complications, such as midline (14\%) and perineal (19\%) wound infection, fistula (4\%), and abscesses (12\%), are the most common complications after TPE.

Preoperative radiotherapy did not lead to an increase in complications, which is in contrast to what has been described by Lopez et al., who identified significant higher complication rates in the irradiated group of patients [27].

Thirty-eight percent of all major complications were related to the construction of the urinary conduit. Houvenaeghel et al. [28] showed similar complications (42\%) related to the construction of a noncontinent urostomy. High complication rates after construction of a Bricker urostomy in irradiated patients often have been reported because of postradiation fibrosis. The Bricker procedure remains the most performed technique for urinary diversion. Other procedures using intestinal segment outside of the radiation field, such as jejunum or colon, have been proposed; however, each have their own related complications. Use of jejunum can lead to metabolic complications and colon conduits are reserved for patients without previous colon resection to prevent absorption problems $[29,30]$. The use of continent pouches is increasingly reported, with related functional advantages leading to improved quality of life but also have $46 \%$ pouch-related complications [30].

For many years, age was considered a contraindication for the performance of TPE. In the present study, patients older than aged 70 years had a similar outcome after TPE compared with younger patients, which is confirmed by other recent studies [20, 22]. Not age but physical condition and comorbidities are considered important criteria in selecting patients for TPE.

As a specialized cancer center, a weekly multidisciplinary meeting is held. All patients with pelvic tumors are presented and the treatment program for each individual patient is discussed and planned.

\section{Conclusions}

Total pelvic exenteration is accompanied with considerable morbidity; however, good local control and acceptable overall survival justifies the use of this extensive surgical technique in selected patients with primary locally advanced and recurrent pelvic tumors.

Open Access This article is distributed under the terms of the Creative Commons Attribution Noncommercial License which permits any noncommercial use, distribution, and reproduction in any medium, provided the original author(s) and source are credited.

\section{References}

1. Ferenschild FT, Vermaas M, Nuyttens JJ et al (2006) Value of intraoperative radiotherapy in locally advanced rectal cancer. Dis Colon Rectum 49:1257-1265

2. Vermaas M, Ferenschild FT, Nuyttens JJ et al (2005) Preoperative radiotherapy improves outcome in recurrent rectal cancer. Dis Colon Rectum 48:918-928

3. Vermaas M, Ferenschild FT, Verhoef C et al (2007) Total pelvic exenteration for primary locally advanced and locally recurrent rectal cancer. Eur J Surg Oncol 33:452-458

4. Bricker EM (1950) Bladder substitution after pelvic evisceration. Surg Clin N Am 30:1511

5. Kecmanovic DM, Pavlov MJ, Kovacevic PA, Sepetkovski AV, Ceranic MS, Stamenkovic AB (2003) Management of advanced pelvic cancer by exenteration. Eur J Surg Oncol 29:743-746

6. Lopes A, Poletto AH, Carvalho AL, Ribeiro EA, Granja NM, Rossi BM (2004) Pelvic exenteration and sphincter preservation in the treatment of soft tissue sarcomas. Eur J Surg Oncol 30:972-975

7. de Wilt JH, van Leeuwen DH, Logmans A et al (2007) Pelvic exenteration for primary and recurrent gynaecological malignancies. Eur J Obstet Gynecol Reprod Biol 134:243-248

8. Brunschwig A (1948) Complete excision of pelvic viscera for advanced carcinoma. Cancer 1:177

9. Chen HS, Sheen-Chen SM (2001) Total pelvic exenteration for primary local advanced colorectal cancer. World J Surg 25:15461549

10. Law WL, Chu KW, Choi HK (2000) Total pelvic exenteration for locally advanced rectal cancer. J Am Coll Surg 190:78-83

11. Saito N, Koda K, Takiguchi N et al (2003) Curative surgery for local pelvic recurrence of rectal cancer. Dig Surg 20:192-200

12. Yamada K, Ishizawa T, Niwa K, Chuman Y, Aikou T (2002) Pelvic exenteration and sacral resection for locally advanced primary and recurrent rectal cancer. Dis Colon Rectum 45:10781084

13. Nuyttens JJ, Kolkman-Deurloo IK, Vermaas M et al (2004) Highdose-rate intraoperative radiotherapy for close or positive margins in patients with locally advanced or recurrent rectal cancer. Int J Radiat Oncol Biol Phys 58:106-112

14. Wanebo HJ, Antoniuk P, Koness RJ et al (1999) Pelvic resection of recurrent rectal cancer: technical considerations and outcomes. Dis Colon Rectum 42:1438-1448

15. Quirke P, Durdey P, Dixon MF, Williams NS (1986) Local recurrence of rectal adenocarcinoma due to inadequate surgical 
resection. Histopathological study of lateral tumour spread and surgical excision. Lancet 2:996-999

16. Kaplan EL, Meier P (1958) Nonparametric estimation from incomplete obeservations. J Am Stat Assoc 53:457-481

17. Cox DR (1972) Regression models and life tables. J R Stat Soc B 34:187-220

18. Sharma S, Odunsi K, Driscoll D, Lele S (2005) Pelvic exenterations for gynecological malignancies: twenty-year experience at Roswell Park Cancer Institute. Int J Gynecol Cancer 15:475-482

19. Berek JS, Howe C, Lagasse LD, Hacker NF (2005) Pelvic exenteration for recurrent gynecologic malignancy: survival and morbidity analysis of the 45-year experience at UCLA. Gynecol Oncol 99:153-159

20. Goldberg JM, Piver MS, Hempling RE, Aiduk C, Blumenson L, Recio FO (1998) Improvements in pelvic exenteration: factors responsible for reducing morbidity and mortality. Ann Surg Oncol 5:399-406

21. Parkin DM, Pisani P, Ferlay J (1999) Global cancer statistics. CA Cancer J Clin 49:33-64 1

22. Roos EJ, Van Eijkeren MA, Boon TA, Heintz AP (2005) Pelvic exenteration as treatment of recurrent or advanced gynecologic and urologic cancer. Int J Gynecol Cancer 15:624-629

23. Barakat RR, Goldman NA, Patel DA, Venkatraman ES, Curtin JP (1999) Pelvic exenteration for recurrent endometrial cancer. Gynecol Oncol 75:99-102
24. Lewis JJ, Leung D, Woodruff JM, Brennan MF (1998) Retroperitoneal soft-tissue sarcoma: analysis of 500 patients treated and followed at a single institution. Ann Surg 228:355-365

25. Moriya Y, Akasu T, Fujita S, Yamamoto S (2004) Total pelvic exenteration with distal sacrectomy for fixed recurrent rectal cancer in the pelvis. Dis Colon Rectum 47:2047-2054

26. Jimenez RE, Shoup M, Cohen AM, Paty PB, Guillem J, Wong WD (2003) Contemporary outcomes of total pelvic exenteration in the treatment of colorectal cancer. Dis Colon Rectum 46:16191625

27. Lopez MJ, Standiford SB, Skibba JL (1994) Total pelvic exenteration. A 50-year experience at the Ellis Fischel Cancer Center. Arch Surg 129:390-396

28. Houvenaeghel G, Moutardier V, Karsenty G et al (2004) Major complications of urinary diversion after pelvic exenteration for gynecologic malignancies: a 23-year mono-institutional experience in 124 patients. Gynecol Oncol 92(2):680-683

29. Mills RD, Studer UE (1999) Metabolic consequences of continent urinary diversion. J Urol 161:1057-1066

30. Karsenty G, Moutardier V, Lelong B et al (2005) Long-term follow-up of continent urinary diversion after pelvic exenteration for gynecologic malignancies. Gynecol Oncol 97:524-528 